Richard B. Lipton

Joshua Liberman

F. Michael Cutrer

Peter J. Goadsby

Michel Ferrari

David W. Dodick

Douglas McCrory

Paul Williams

\section{Treatment preferences and the selection of acute migraine medications: results from a population-based survey}

Received: 25 November 2003

Accepted in present form: 14 January 2004

R.B. Lipton ( $\square)$

Departments of Neurology, Epidemiology and Population Health,

Albert Einstein College of Medicine,

1300 Morris Park Avenue (Russo-3rd Floor),

Bronx, NY 10461, USA

e-mail: rlipton@aecom.yu.edu

Tel.: 718-430-3886

Fax: 718-430-3857

J. Liberman

IMR, an Advance PCS Company,

Hunt Valley, USA

F.M. Cutrer

Mayo Clinic, Rochester, USA

P.J. Goadsby

Institute of Neurology

The National Hospital for Neurology and

Neurosurgery,

London, UK

M. Ferrari

Department of Neurology,

Leiden University Medical Centre,

Leiden, The Netherlands

D.W. Dodick

Department of Neurology,

Mayo Clinic, Scottsdale, AZ, USA

D. McCrory

Duke University Center for Clinical Health,

Policy Research,

Durham, USA

P. Williams

PAREXEL International,

Uxbridge, Middlesex, UK

\begin{abstract}
Seven oral triptans are available for treating acute migraine. We surveyed US migraine sufferers on the relative importance of treatment attributes for choosing among oral triptans. A multiattribute decision model was used to combine data on the relative importance of efficacy, consistency, and tolerability of acute treatment (determined by 206 triptan-experienced and 209 triptannaive subjects) with data on the performance of the triptans across the attributes (derived from a recent meta-analysis). Efficacy was considered significantly more important than tolerability and consistency: tolerability was significantly more important than consistency for triptan-naive but not triptan-experienced subjects. The multiattribute decision model found that almotriptan, eletriptan, and rizatriptan were significantly closer to the hypothetical ideal triptan than the reference product, sumatriptan $100 \mathrm{mg}$, for both triptan-naive and triptan-experienced migraine sufferers. Almotriptan, eletriptan, and rizatriptan were the preferred triptans selected on the basis of patients' own priorities and product performance data.
\end{abstract}

Key words Meta-analysis • Migraine $\cdot$ Surveys $\cdot$ Treatment attributes - TRIPSTAR - Triptans 


\section{Introduction}

According to the American Migraine Study II, a large population-based survey, approximately 28 million Americans suffer from migraine [1]. Migraine is more prevalent in whites than in other ethnic groups, more common in lowerincome than higher-income groups, and has a peak prevalence from ages 25 to 55 years, the most productive years of an individual's life [1].

Migraine is characterized by severe pain and often by headache-related disability, which causes absenteeism as well as reduced productivity, imparting a substantial diseaserelated burden on the individual and economic burden on society. Of all migraineurs in the American Migraine Study II, 53\% reported severe impairment of their daily activities or a need for bed rest with severe headaches [1]. Moreover, the Global Burden of Disease Study, sponsored by the World Health Organization, ranked the disability associated with severe migraine among the world's 20 most disabling chronic disorders [2] and lost labor costs in the United States have been estimated at $\$ 13$ billion per year [3].

Headache-related disability is an important target for migraine therapy. Yet despite an increase in the diagnosis of migraine over the past decade, the majority of migraineurs remain undiagnosed [4] and the proportion of migraine sufferers treating with a prescription medication only increased modestly from $37 \%$ in 1989 to $41 \%$ in 1999 [4]. Factors contributing to these low rates of diagnosis and treatment include failure to consult a physician, poor patient-physician communication, and patient reluctance to take a prescription migraine medication due to safety concerns [4].

Treatment patterns, reported in a more recent survey, show that about half of migraineurs use over-the-counter medications exclusively, $21 \%$ use prescription medications exclusively, and $23 \%$ use both [5]. In the prescription-only group, 36\% used butalbital-caffeine combinations and only $18 \%$ used triptans [5]. This underuse of triptans prevails despite American Academy of Neurology guidelines recommending triptans as the treatment of choice for patients with moderate-to-severe migraine and for those with migraine of any severity who have not previously responded to nonspecific medications (e.g. non-steroidal antiinflammatory drugs [NSAIDS], analgesics) [6].

Seven orally administered triptans are now available to treat patients with acute migraine: almotriptan, eletriptan, frovatriptan, naratriptan, sumatriptan, rizatriptan, and zolmitriptan. A recent meta-analysis evaluated 6 of these triptans in more than 24000 patients in 53 published and unpublished controlled trials; the conclusions were that these agents differed with respect to efficacy, consistency of effect, rate of recurrence, and tolerability, and that these differences were clinically relevant to the individual $[7,8]$. Matching triptans to the needs of individual patients is a challenging task. Indeed, a study of neurologists' learning needs (when only 4 of the 7 currently available triptans were on the market) found that while all felt that they used evidence-based medicine in their daily practice, there was considerable uncertainty about how to appraise the triptans and select which to prescribe [9].

This issue can be framed as a multiattribute decision problem, in which a decision maker must select from among competing alternatives on the basis of multiple criteria and points of view [10]. Multiattribute decision-making (MADM) methods, frequently used in public sector and business settings [11-13], have only rarely been applied to the issue of selecting medical interventions, but recent examples include the Star Systems, used to aid physicians in choosing among antiepileptic drugs [14], and an evaluation of the performance of imaging techniques for breast cancer [15].

In this article, we first report the relative importance of prespecified treatment attributes for selecting an oral triptan, from the perspectives of 2 groups of headache sufferers. The first group comprised triptan users, while the second group consisted of triptan-naive subjects with high levels of disability (Migraine disability assessment [MIDAS] grades III or IV), who might be considered to be candidates for triptans. We then combine these data with information on the relative performance of the triptans as reported in a recent meta-analysis [7, 8] using a multiattribute decision model to identify the preferred triptans.

\section{Methods}

Population survey

Data on the relative importance to migraineurs of selected triptan treatment attributes were collected in a cross-sectional population survey. A random sample of us households with a telephone were contacted, and a computer-assisted telephone interview (CATI) was conducted among eligible and willing headache sufferers between the ages of 18 and 60 years. We administered both a diagnostic interview and the MIDAS questionnaire by phone and after eliminating the ineligible candidates, selected 2 groups: the triptanexperienced group $(n=206)$ comprised subjects who treated at least one headache with a triptan in the past 12 months, and the triptannaive group $(n=209)$ consisted of individuals who never used a triptan to treat a headache but who reported significant headacherelated disability, as measured by MIDAS scores of III or IV [16].

Respondents were questioned about their medical and treatment history and their headache characteristics. They were asked to evaluate the relative importance - in selecting "a new treatment for your headache" - of a prespecified set of treatment attributes, which were taken from those used to evaluate oral triptans in the meta-analysis, thus ensuring the availability of controlled clinical trial data for subsequent comparison [7]. Queries were restricted to the relative importance of treatment attributes; migraineurs were not asked to rate the attributes of specific drugs. Treatment attributes were arranged into a hierarchy of 3 top-level attributes (efficacy, consis- 


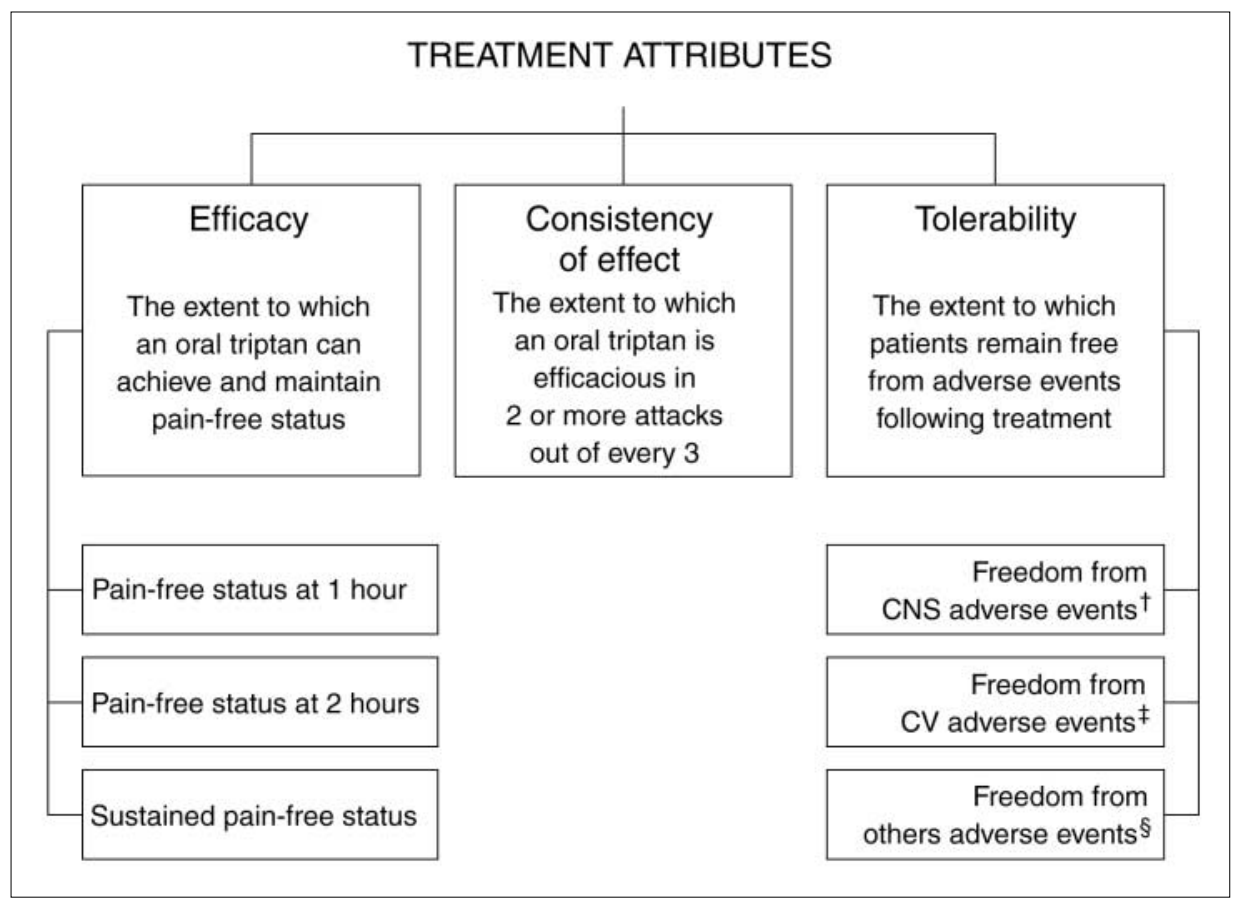

Fig. 1 Treatment attributes that survey participants were asked to evaluate regarding the choice of a headache medication. Sustained pain-free status was defined as no pain 2 hours after dosing, with no recurrence of moderate or severe headache, and no use of any rescue headache medication 2-24 hours after dosing. ${ }^{\dagger}$ Central nervous system $(C N S)$ adverse events included sedation and dizziness. ‡Cardiovascular $(C V)$ adverse events included chest pressure, chest pain and palpitations. §Other adverse events included paresthesias, flushing and limb heaviness

tency of effect, and tolerability) and 2 sets of lower-level efficacy and tolerability attributes (Fig. 1). The lower-level efficacy attributes were pain-free status at 1 hour, pain-free status at 2 hours, and sustained pain-free status (no pain 2 hours postdose, without recurrence of moderate or severe headache or use of rescue headache medication 2-24 hours postdose). The lower-level tolerability attributes were freedom from cardiovascular (CV) adverse events (side effects such as chest pressure or pain), freedom from central nervous system (CNS) adverse events (side effects such as such as sleepiness or dizziness), and freedom from other adverse events (Fig. 1).

The relative importance of the attributes was assessed in a series of pair-wise comparisons, and the ratings from each participant were transformed into importance weights using a matrixmultiplication algorithm [17] and scaled to sum to $100 \%$ within each group. Confidence intervals for the mean importance weights for the entire sample (separately for the triptan and MIDAS groups) were estimated in a nonparametric bootstrapping exercise with 10000 resamples [18].

\section{Triptan performance}

Performance data on the efficacy and tolerability attributes of 6 oral triptans were obtained from a meta-analysis of 53 published and unpublished, randomized, double-blind, placebo- or active-controlled migraine treatment trials in more than 24000 patients $[7,8]$.

Multiattribute decision modelling

All MADM problems can be expressed as a matrix. In the MADM matrix used here, triptan treatment attributes such as efficacy, consis- tency of effect, and tolerability were plotted against 6 triptan alternatives. The performance of the triptans on these attributes, and weights reflecting the relative importance of the attributes, were combined according to prespecified decision rules to generate an overall evaluation of the desirability of, or preference for, each of the triptans. The attribute importance weights from the survey were combined with the performance data from the meta-analysis using a modified version of the MADM model Technique for Order Preference by Similarity to Ideal Solution (TOPSIS) [19, 20]. In the TOPSIS model, data are first standardized to take into account differences in measurement units. Then, in keeping with Zeleny's axiom of human choice ("Alternatives closer to the ideal are preferred to those further away. To be as close as possible to the perceived ideal is the rationale of human choice.") [21], the model identifies a hypothetical "ideal" triptan: a composite that, if it really existed, would perform best on every attribute. The definition of the "ideal" for the purpose of this model is "best achievable with current technology" or optimal. Next, the model measures the distance of each of the 6 real triptans from the hypothetical ideal (by calculating the weighted Euclidean distance) [13]. These distance measures are scaled such that 0 is identical to the "anti-ideal" (i.e. the worst possible) and $100 \%$ is identical to the ideal (the best possible), while intermediate values measure similarity to the ideal triptan, providing the basis for comparing the triptans.

The models were run twice, first using absolute, and then placebo-corrected efficacy and consistency data (the tolerability data were always placebo-corrected). A correction was introduced to remove the effect of sustained pain-free rates for placebo that were significantly higher in the almotriptan studies and significantly lower in the eletriptan studies [22]. Because there were no consistency data for zolmitriptan, a consistency level equal to the mean of all the other products was assumed.

To account for the variance in the meta-analysis results and in the survey data, $95 \%$ confidence intervals for the similarity scores were estimated by incorporating probabilistic uncertainty analysis [23] into 
the bootstrapping. As in the meta-analysis, statistical significance was assessed using sumatriptan $100 \mathrm{mg}$ as the reference product. In each of the 10000 uncertainty analysis iterations, the similarity score of each product was compared to that of the reference product, and triptans were considered significantly superior to sumatriptan $100 \mathrm{mg}$ if they were closer to the ideal on $\geq 95 \%$ of the resamples.

\section{Results}

The participants in both the high disability triptan-naive group and the triptan group were predominantly white women in their late 30s (Table 1). The time since initial diagnosis was significantly longer for the triptan-experienced group than for the triptan-naive group (16 and 12 years, respectively; MannWhitney $\mathrm{z}=2.42, p<0.02)$. Subjects in the triptan-experienced group were more likely to describe the majority of their headaches as moderate or severe in intensity $\left(\chi^{2}=7.69\right.$, $p<0.01)$, unilateral $\left(\chi^{2}=15.52, p<0.001\right)$, and involving changes in vision $\left(\chi^{2}=6.79, p<0.01\right)$, nausea or vomiting $\left(\chi^{2}=28.64, p<0.0001\right)$, and phono- or photophobia $\left(\chi^{2}=11.05\right.$, $p<0.001)$. Only a small percentage of participants in either group felt completely satisfied with the usual treatment (Table 1). Respondents were much more likely to be dissatisfied with an efficacy parameter than with tolerability.

\section{Relative importance of triptan treatment attributes}

Table 2 gives the mean importance weights (and their bootstrap $95 \%$ confidence limits) for the top-level efficacy and tolerability attributes, reported separately for the study groups. Efficacy attributes were considered to be the most important of the top-level attributes by both the triptan-naive group and the triptan-experienced group. For the triptannaive group, but not the triptan-experienced group, tolerability attributes were significantly more important than consistency of effect. This pattern (efficacy>tolerability $>$ consistency for the triptan-naive group; efficacy $>$ [tolerability $\approx$ consistency] for the triptan-experienced group) was consistent across subgroups defined according to personal and headache characteristics set out in Table 1 (data not shown).

Table 1 Characteristics of survey respondents

Women, $\mathrm{n}(\%)$
Men, $\mathrm{n}(\%)$
Age, years ${ }^{\mathrm{a}}$
Caucasian-white race, $\mathrm{n}(\%)$
Age at diagnosis, years ${ }^{\mathrm{a}}$
Years since diagnosis
Headache days in previous 3 months ${ }^{\mathrm{b}}$
$\geq 50 \%$ of headaches of moderate-severe intensity, $\mathrm{n}(\%)$
Headache duration, with treatment, $\mathrm{h}^{\mathrm{b}}$
Headache symptoms, $\mathrm{n}(\%)$
Changes in vision on $\geq 50 \%$ of occasions
Unilateral headache on $\geq 50 \%$ of occasions
Nausea or vomiting on $\geq 50 \%$ of occasions
Phono- or photophobia on $\geq 50 \%$ of occasions
Headache-related disability, MIDAS grades III or IV
Satisfaction with "usual treatment", $\mathrm{n}(\%)$
Completely satisfied
Inadequate level of pain relief
Relief takes too long
Doesn't always work
Recurrence
Adverse events
Other/not stated

Triptan-experienced group $(n=206)$

$\begin{array}{rrrr}175 & (84) & 186 & (90) \\ 34 & (16) & 20 & (16) \\ 37 & (11.2) & 39 & (10.6) \\ 166 & (79) & 176 & (85) \\ 22 & (9.9) & 22 & (10.6) \\ 12 & (6-21) & 16 & (8-25) \\ 8 & (5-20) & 8 & (3-20) \\ 166 & (79) & 184 & (89) \\ 3 & (1-12) & 4 & (1-24) \\ & & & \\ 77 & (37) & 102 & (50) \\ 111 & (53) & 148 & (72) \\ 95 & (45) & 147 & (71) \\ 171 & (82) & 191 & (93) \\ 209 & (100) & 118 & (57) \\ & & & \\ 18 & (9) & 26 & (13) \\ 36 & (17) & 16 & (8) \\ 47 & (22) & 31 & (15) \\ 46 & (22) & 51 & (25) \\ 50 & (24) & 42 & (20) \\ 7 & (3) & 18 & (9) \\ 5 & (3) & 22 & (10)\end{array}$

MIDAS, migraine disability assessment

a Values are mean (SD)

$\mathrm{b}$ Values are median (range) 
Table 2 Relative importance of the triptan treatment attributes. Values are mean importance weights (bootstrap 95\% confidence intervals)

\begin{tabular}{lrr}
\hline Attribute & $\begin{array}{r}\text { Triptan-naive group } \\
(\mathrm{n}=209)\end{array}$ & $\begin{array}{r}\text { Triptan-experienced group } \\
(\mathrm{n}=206)\end{array}$ \\
\hline Top-level & & \\
$\quad$ Efficacy & $45(41-51)$ & $49(41-52)$ \\
$\quad$ Consistency of effect & $23(18-24)$ & $28-31)$ \\
$\quad$ Tolerability & $32(29-40)$ & $23(22-29)$ \\
Efficacy & $58(52-63)$ & $56(55-62)$ \\
$\quad$ Pain-free status at $1 \mathrm{~h}$ & $13(11-16)$ & $14-10-15)$ \\
$\quad$ Pain-free status at $2 \mathrm{~h}$ & $29(25-34)$ & $30(31-40)$ \\
$\quad$ Sustained pain-free status & & \\
Tolerability & $20(17-28)$ & $20 \quad(15-27)$ \\
$\quad$ Freedom from CNS adverse events & $47(41-55)$ & $54(47-65)$ \\
$\quad$ Freedom from CV adverse events & $34(25-36)$ & $27(19-31)$ \\
$\quad$ Freedom from other adverse events & & \\
\hline
\end{tabular}

$C N S$, central nervous system; $C V$, cardiovascular

No differences were observed between the triptannaive group and triptan-experienced groups with respect to the relative importance ascribed to the 3 efficacy attributes (Table 2). According to the respondents, pain-free status at 1 hour was the most important of the efficacy attributes, followed by sustained pain-free status. This pattern of results (pain-free status at $1 \mathrm{~h}>$ sustained pain-free status $>$ pain-free status at $2 \mathrm{~h}$ ) was observed consistently across subgroups defined according to the personal and headache characteristics set out in Table 1 .

Similarly, no differences were observed between the groups for the relative importance assigned to the 3 tolerability attributes (Table 2). About half the total weight ascribed to tolerability was accounted for by the most important tolerability attribute, freedom from CV adverse events. The order of importance (freedom from $\mathrm{CV}$ adverse events $>$ other adverse events > CNS adverse events) was consistent across subgroups defined according to personal and headache characteristics (data not shown).

Similarity to the hypothetical ideal triptan

Importance weights elicited from survey participants were combined with clinical trial data from the meta-analysis in a TOPSIS model. Mean similarity to the ideal scores, $95 \%$ confidence limits, and statistical significance levels were estimated for each product as described previously. At the group level of analysis, 3 oral triptans (almotriptan, eletriptan, and rizatriptan) were significantly closer to the hypothetical ideal triptan than was the reference product,

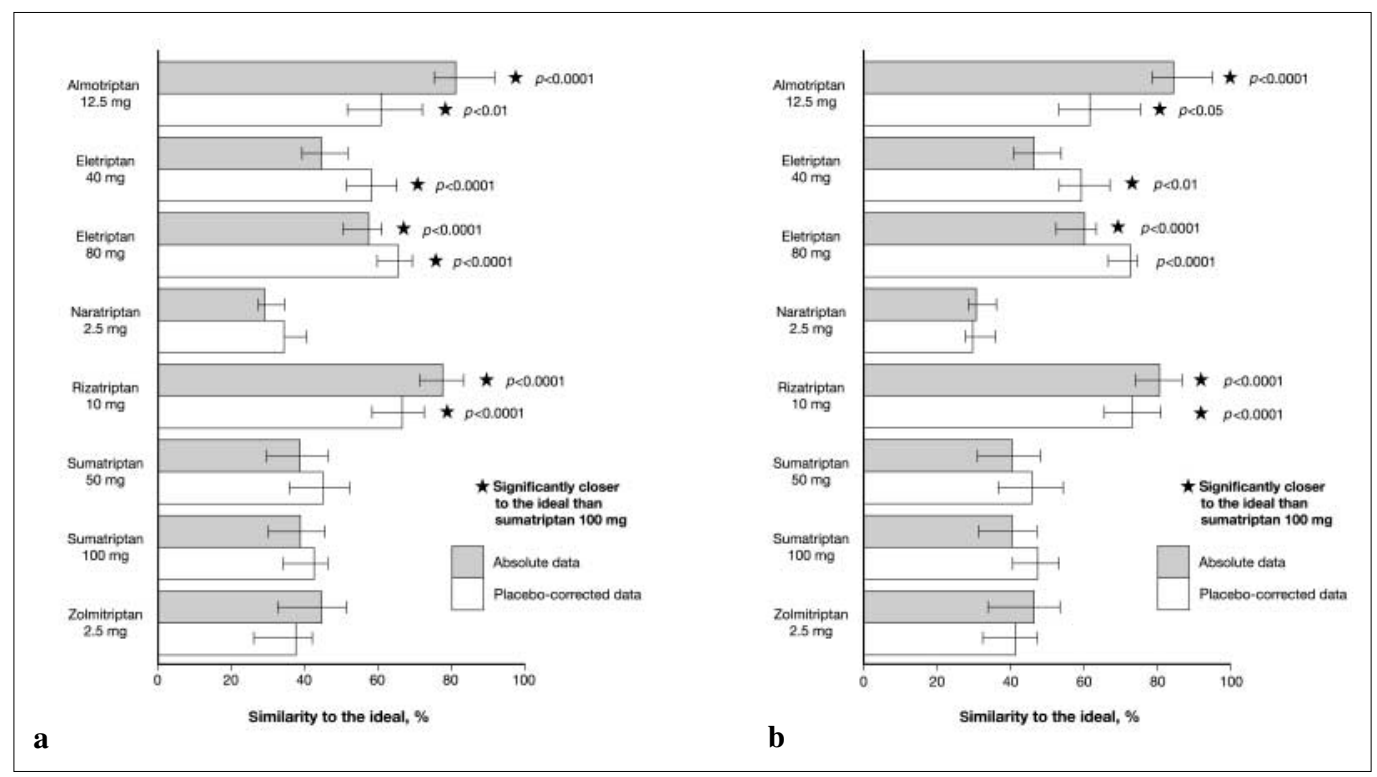

Fig. 2a, b Similarity of triptans avaiable for oral use to the ideal triptan. a Triptan-naive group. b Triptan-experienced group. Values are mean $(95 \% \mathrm{CI})$ 
sumatriptan $100 \mathrm{mg}$ (Fig. 2). This finding was true for both study groups. The 40-mg dose of eletriptan was significantly closer to the ideal than sumatriptan $100 \mathrm{mg}$ in the triptan-naive group when placebo-corrected or absolute data were used, but significantly closer in the triptan-experienced group only when placebo-corrected data were used. Almotriptan $12.5 \mathrm{mg}$, eletriptan $80 \mathrm{mg}$, and rizatriptan $10 \mathrm{mg}$ were significantly better than sumatriptan 100 $\mathrm{mg}$ regardless of whether absolute or placebo-corrected data were used. At the individual level of analysis, almotriptan, eletriptan, and rizatriptan constituted the "top 3 " closest to the hypothetical ideal for $77 \%-78 \%$ of the triptan-naive group and $85 \%-86 \%$ of the triptan-experienced group of migraine sufferers, based on the importance weights elicited from the participants.

\section{Discussion}

According to the survey respondents, efficacy was significantly more important than tolerability or consistency of effect in selecting an oral triptan. For the triptan-naive (high MIDAS score) group, tolerability was significantly more important than consistency, while the triptan-experienced group attached equal importance to tolerability and consistency. Pain-free status at 1 hour and freedom from $\mathrm{CV}$ adverse events were considered to be the most important lower-level attributes for both groups. This pattern was consistent across subgroups defined according to personal and headache characteristics.

Converging evidence indicates that complete and rapid pain relief without recurrence is the most important treatment attribute for migraine sufferers, as is also observed in the present survey. According to 648 migraineurs participating in a placebo-controlled sumatriptan clinical trial [24], the 4 most important attributes of migraine therapy were "how well it works", "how safe it is", "how fast it works", and "side effects"; the least important attribute was "cost of drug". In a population-based telephone survey, 688 migraineurs reported that complete pain relief $(87 \%)$, no recurrence $(86 \%)$, rapid onset $(83 \%)$, and no side effects $(79 \%)$ were the most important attributes of acute treatment. Of these patients, $29 \%$ were very satisfied with their acute migraine therapy, $48 \%$ were somewhat satisfied, $7 \%$ were neither satisfied nor dissatisfied, $9 \%$ were somewhat dissatisfied and $7 \%$ were very dissatisfied $[25,26]$. Reasons for dissatisfaction were pain relief taking too long $(87 \%)$, incomplete pain relief $(84 \%)$, inconsistent relief $(84 \%)$, headache recurrence $(71 \%)$, and too many side effects (35\%). These results are supported by post-hoc analyses of clinical trials showing that fast and complete pain relief predicts satisfaction and health-related quality-of-life [27-29].
Weightings of the treatment attributes derived from migraine sufferers in the present study can be compared with those of neurologists and primary care physicians (PCPs) who were surveyed as part of the TRIPSTAR project $[30,31]$. Both physicians and migraine sufferers rated efficacy as the most important top-level attribute. Like the triptan-naive (high MIDAS score) respondents themselves, physicians rated tolerability as more important than consistency for their triptan-naive patients. Physicians and migraine sufferers agreed that freedom from $\mathrm{CV}$ adverse events was the most important lower-level tolerability attribute but disagreed as to the most important lower-level efficacy attribute. Migraine sufferers weighted pain-free status at 1 hour as more important than sustained pain-free status, whereas physicians believed sustained pain-free status was more important.

The attribute importance weights obtained from the migraine sufferers were combined in a TOPSIS model with clinical trial data from a meta-analysis [7, 8]. Three triptans (almotriptan, eletriptan, and rizatriptan) were consistently found to be significantly closer to the hypothetical ideal of the decision model than was the reference product, sumatriptan $100 \mathrm{mg}$. This was true for both the triptannaive (high MIDAS score) group and the triptan-experienced group, using either absolute or placebo-corrected data at both the group and individual analysis levels.

These results must be assessed in the context of limitations imposed by the multiattribute decision-making model, its inherent assumptions and its structure, as well as the data used in the models. The TOPSIS model used here was chosen not only because of its intuitive appeal ("similarity to the ideal"), but also because it makes no distributional assumptions beyond preference independence and additivity, which are common to all decision models. An important model-related issue, however, is that the outcome of a model depends on the inputs. In the present context, a different selection of treatment attributes may well have led to a different set of results. For example, if a "familiarity of use" attribute had been included, this would have influenced the model in favor of the older triptans. Also, the model used for the TRIPSTAR project did not include any subjective attributes, and only treatment-related attributes for which robust data are available were included, i.e. those studied in the recent meta-analysis [7, 8].

The 3 oral triptans that emerged as superior in this study also constituted the preferred subset based on the surveys of US neurologists and PCPs carried out as part of the TRIPSTAR project [30, 31]. Furthermore, attribute importance weights elicited in response to a case-history presented at a symposium yielded the same set of preferred products (almotriptan, eletriptan, and rizatriptan) when combined with the meta-analysis data in a TOPSIS model 
$[8,32]$. A reasonable degree of convergent validity for the superiority of these 3 agents (based on the treatment attributes included in the model) can therefore be said to exist.

The explanation for the consistency of this finding lies in the dominance hierarchy found in the meta-analysis results. As McCrory [33] pointed out, all other triptans are dominated (in a decision-analytic sense) by one or more of these 3 products, and within this subset of preferred products (almotriptan, eletriptan, rizatriptan), no one dominates any of the others. The superiority of this trio was further supported in a TOPSIS model which used computer-generated attribute importance weights representing the entire range of possible values for the relative importance of the treatment attributes [34].

Multiattribute decision-making models such as TOPSIS have a potential role in improving the match between patients' needs and treatment choice. Computer-based clinical decision support systems are becoming increasingly prevalent, ranging in complexity from simple patient recall systems through drug-dosing calculators, to complex diagnostic tools linked to electronic medical records [35]. A review of meta-analyses of trials of the effectiveness of such systems concluded that they may provide significant benefits in the process of care [35]. A model of the kind used in the TRIPSTAR project has considerable potential as a decision-support tool in the management of patients with migraine, as it provides a convenient way of incorporating migraine sufferers' own viewpoints with objective data on the performance of the oral triptans.

A recent study by Lipton et al. [5, 36] revealed that while $48 \%$ of US migraineurs consulted with physicians over the last year, $21 \%$ had lapsed from care, many believing that there was nothing a physician could do to help them. Tailoring treatment to individuals' priorities may increase the level of satisfaction among migraineurs and improve adherence to treatment. While the results of the TRIPSTAR project should not influence physicians to change the triptan therapy of migraineurs who are satisfied with their current treatment, the evidence nevertheless suggests that selection from the subset of preferred triptans (almotriptan, eletriptan, and rizatriptan) is a useful starting point for newly diagnosed migraineurs and for those who are dissatisfied with their current treatment.

Acknowledgement This project was supported by a grant from Pharmacia Corporation.

\section{References}

1. Lipton RB, Stewart WF, Diamond S, Diamond ML, Reed M (2001)

Prevalence and burden of migraine in the United States: data from the American Migraine Study II. Headache 41(7):646-657

2. Menken M, Munsat TL, Toole JF (2000) The global burden of disease study: implications for neurology. Arch Neurol 57(3):418-420

3. Hu XH, Markson LE, Lipton RB, Stewart WF, Berger ML (1999) Burden of migraine in the United States: disability and economic costs. Arch Intern Med 159(8):813-818

4. Lipton RB, Diamond S, Reed M, Diamond ML, Stewart WF (2001) Migraine diagnosis and treatment: results from the American Migraine Study II. Headache 41(7):638-645

5. Lipton RB, Scher AI, Steiner TJ, Bigal ME, Kolodner K, Liberman JN, Stewart WF (2003) Patterns of health care utilization for migraine in England and in the United States. Neurology 60(3):441-448
6. Silberstein SD (2000) Practice parameter: evidence-based guidelines for migraine headache (an evidence-based review): report of the Quality Standards Subcommittee of the American Academy of Neurology. Neurology 55(6):754-762

7. Ferrari MD, Roon KI, Lipton RB, Goadsby PJ (2001) Oral triptans (serotonin $5-\mathrm{HT}_{1 \mathrm{~B} / 1 \mathrm{D}}$ agonists) in acute migraine treatment: a metaanalysis of 53 trials. Lancet 358:1668-1675

8. Ferrari MD (2002) TRIPSTAR: A comprehensive patient-based approach to compare triptans. Headache 42[Suppl 1]:S18-S25

9. Purdy RA (2000) Evidence-based migraine therapy: learning needs and knowledge assessment. Cephalalgia 20[Suppl 2]:5-9

10. von Winterfeldt D, Edwards W (1987) Decision analysis and behavioral research. Cambridge University Press, Cambridge
11. Barnes JA, Rutherford GS (1997) Analysis of the initial application of the State of Washington highway mobility project: ranking procedure and recommended revisions for the upcoming biennium. Washington State Department of Transportation Report Number WA-RD 428.1. Abstract available at http://www.wsdot.wa.gov/ research/onepages/WA-RD4281.htm (accessed 17 June 2004)

12. Jackson HV (1999) A structured approach for classifying and prioritizing product requirements. $\mathrm{PhD}$ thesis, North Carolina State University. Available at http://www.lib.ncsu.edu/ etd/public/etd 354921279912131/ etd.pdf (accessed 17 June 2004)

13. Deng H, Yeh CH, Willis RJ (2000) Inter-company comparison using modified TOPSIS with objective weights. Comput Operations Res 27:963-973

14. Brodie MJ, Kwan P (2001) The Star Systems: overview and use in determining antiepileptic drug choice. CNS Drugs 15(1):1-12 
15. Azar F (2000) Multi-attribute decision making: use of three scoring methods to compare the performance of imaging techniques for breast cancer detection. Avaiable at http://www.cis.upenn.edu/ fredazar/pu blications/pdf/fredazar_techreport_M S-CIS-00-10.PDF (accessed 17 June 2004)

16. Stewart WF, Lipton RB, Dowson AJ, Sawyer J (2001) Development and testing of the Migraine Disability Assessment (MIDAS) questionnaire to assess headache-related disability. Neurology 56[6 Suppl 1]:S20-S28

17. Dolan JG (1989) Medical decision making using the analytic hierarchy process: choice of initial antimicrobial therapy for acute pyelonephritis. Med Decis Making 9(1):51-56

18. Mooney CZ, Duval RD (1993) Bootstrapping: a nonparametric approach to statistical interference. Sage, Newbury Park

19. Hwang CL, Yoon KP (1981) Multiattribute decision making: methods and applications. Springer, Berlin Heidelberg New York, pp 128-140

20. Yoon KP, Hwang CL (1995) Multiple attribute decision making: an introduction. Sage, Thousand Oaks, pp 38-40

21. Zeleny M (1982) Multiple criteria decision making. McGraw Hill, New York

22. Roon KI, Lipton R, Goadsby PJ, Ferrari M (2001) Placebo in triptan trials: efficacy, tolerability and consistency. Cephalalgia 21:405-432
23. Baltussen RM, Hutubessy RC, Evans DB, Murray CJ (2001) Uncertainty in cost-effectiveness analyses: probabilistic uncertainty analysis and stochastic league tables. GPE Discussion Paper Series, no. 34. World Health Organization, Geneva Available at http://www3.who.int/whosis/statistics/discussion_papers/pdf/paper34.pd f (accessed 17 June 2004)

24. Luciani RJ, Osterhaus JT, Gutterman DL (1995) Patient preferences for migraine therapy: subcutaneous sumatriptan compared with other medications. J Fam Pract 41:147-152

25. Lipton RB, Stewart WF (1999) Acute migraine therapy: do doctors understand what patients with migraine want from therapy? Headache 39[Suppl 2]:S20-S26

26. Lipton RB, Hamelsky SW, Dayno JM (2002) What do patients with migraine want from acute migraine treatment? Headache 42[Suppl 1]:S3-S9

27. Davies GM, Santanello N, Lipton R (2000) Determinants of patient satisfaction with migraine therapy. Cephalalgia 20(6):554-560

28. Santanello NC, Davies G, Allen C, Kramer M, Lipton R (2002) Determinants of migraine-specific quality of life. Cephalalgia 22(8):680-685

29. Loder E, Brandes JL, Silberstein S, Skobieranda F, Bohidar N, Wang L, Boyle D, Kolodny A, Guerra F, Santanello N, Johnson-Pratt L (2001) Preference comparison of rizatriptan ODT 10-mg and sumatriptan 50-mg tablet in migraine. Headache 41(8):745-753
30. Dodick D, Cutrer FM, Ferrari M, Goadsby PJ, Lipton RB, McCrory DC, Williams P (2002) Prioritization of treatment attributes in selecting an oral triptan: a survey of US neurologists. Headache 42:392

31. Cutrer FM, Goadsby PJ, Ferrari M, Lipton R, Dodick D, McCrory D (2002) Prioritization of treatment attributes in selecting an oral triptan: a survey of U.S. primary care physicians. Headache 42:392-393

32. Dodick DW, Lipton RB, Goadsby PJ, McCrory D, Ferrari M, Williams P, Cutrer FM (2002) Prioritizing triptan treatment attributes: a pilot study. Neurology 58[Suppl 3]:A129

33. McCrory D (2002) Dominance relationships in the triptan meta-analysis. In: 14th Annual Migraine Trust International Symposium, September 23-26, London, UK

34. Ferrari MD (2002) Tripstar: a comprehensive patient-based approach to compare triptans. Headache 42[Suppl 1]:18-25

35. Trowbridge R, Weingarten S (2001) Clinical decision support systems. In: Making health care safer: a critical analysis of patient safety practices. Evidence Report/Technology Assessment, no. 43. Agency for Healthcare Research and Quality, Rockville (AHRC publication no. 01E058, Chapt. 53). Available at http://www.ahcpr.gov/clinic/ptsafety/c hap53.htm (accessed 17 June 2004)

36. Lipton RB, Scher AI, Kolodner K, Liberman J, Steiner TJ, Stewart WF (2002) Migraine in the United States: epidemiology and patterns of health care use. Neurology 58(6):885-894 